\title{
A LOW EMITTANCE LATTICE FOR THE NSLS X-RAY RING *
}

\section{J. Safranek, National Synchrotron Light Source, Brookhaven National Laboratory, Upton, NY 11973}

Abstract

A new high-brightness lattice for the NSLS X-Ray Ring is presented. The horizontal emittance of the X-Ray Ring can be reduced by a factor of two to three by allowing nonzero dispersion in the insertion device straight sections and by better controlling the periodicity of the dispersion. Beam was stored in a low emittance lattice during machine studies. The dispersion in the operations lattice lacks the 8fold periodicity of the magnet structure, because the orbit is offset in the sextupoles. During machine studies the focusing effect of the sextupoles was compensated using trim supplies on the quadrupoles in the dispersive straight sections. Measured results will be presented. A discussion of the hardware upgrade requirements that would be necessary to run the low emittance lattice in operations is also included.

\section{INTRODUCTION}

In the past the focusing gradients of the NSLS X-Ray Ring magnets were not known to high precision. For example, figure 1 shows the measured horizontal dispersion compared to the accepted model [1] of the ring optics. The work described in references [2] and [3] has enabled us to precisely determine the true focusing gradients in all the $\mathrm{X}$-Ray Ring magnets. With this improved understanding of the ring optics we are now better able to control the horizontal dispersion and beta function. We can now correct for local gradient errors, arising from displaced orbits in sextupoles, that break the periodicity of the dispersion, and we can modify the quadrupole family strengths in order to minimize the horizontal emittance.

The present X-Ray Ring optics give a horizontal emittance of $113 \mathrm{~nm}^{*}$ rad. With minimal hardware changes, this emittance can be reduced to $49 \mathrm{~nm}^{*}$ rad. The emittance could be further reduced to $38 \mathrm{~nm}^{*}$ rad, but this would require replacing the sextupoles used for correcting the vertical chromaticity. This reduced horizontal emittance electron beam would produce a brighter $\mathrm{x}$-ray beam for the NSLS users, adding to other recent increases in the X-Ray Ring brightness [4].

"Work performed under the auspices of the U.S. Department of Energy

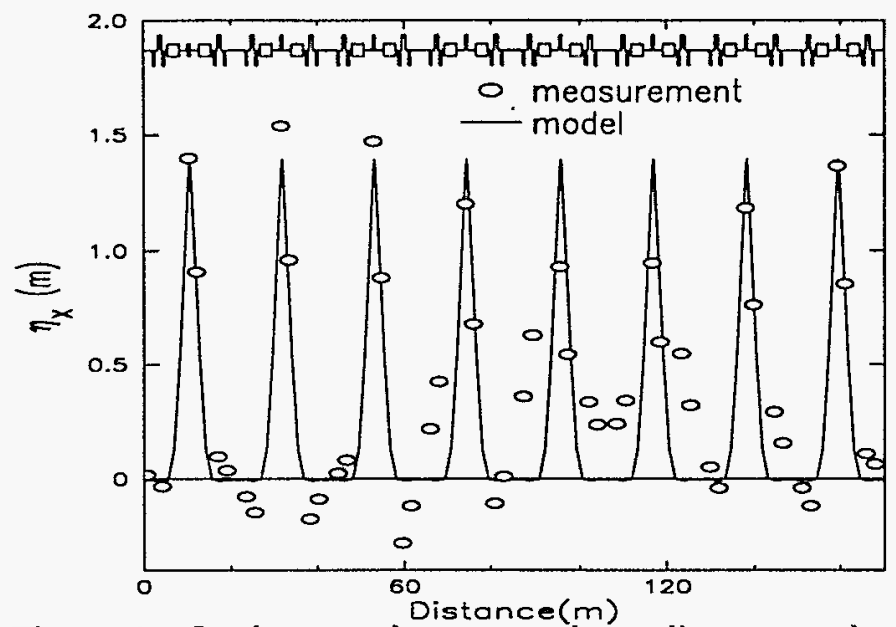

Figure. 1. In the past there was a large discrepancy between the measured and model dispersion, due primarily to focusing in the sextupole magnets.

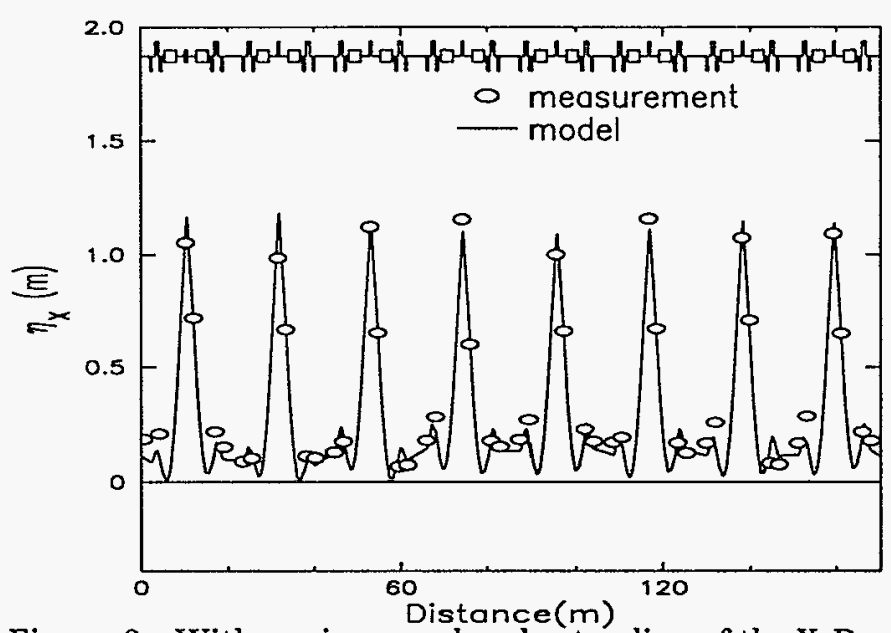

Figure. 2. With our improved understanding of the X-Ray Ring lattice we can adjust the quadrupoles to give $\eta_{x}$ the design periodicity and reduce the emittance.

\section{MINIMUM X-RAY RING EMITTANCE}

\section{A. Minimum theoretical emittance}

The emittance, $\epsilon_{x}$, in a storage ring is determined by the horizontal dispersion, $\eta_{x}$, and the horizontal beta function, $\beta_{x}$, in the dipole magnets [5]:

$$
\epsilon_{x}=\frac{C E^{2}}{\rho} \frac{\int_{D}\left(\beta_{x} \eta_{x}^{\prime 2}+2 \alpha_{x} \eta_{x} \eta_{x}^{\prime}+\gamma_{x} \eta_{x}^{2}\right) d s}{\int_{D} d s}
$$

where $C=1.47 \times 10^{-6} \mathrm{~m} * \mathrm{GeV}^{-2}, E$ is the electron energy (2.584 GeV in the X-Ray Ring), $\rho$ is the dipole magnet 
bending radius, $\int_{D}$ is the integral over all the dipole magnets, $\alpha_{x}=-\beta_{x}^{\prime} / 2, \gamma_{x}=\left(1+\alpha_{x}^{2}\right) / \beta_{x}$, and $s$ is the longitudinal position around the ring. The X-Ray Ring has four families of quadrupoles, so the gradients in each quadrupole family can be varied to minimize the emittance. The gradients to obtain the minimum emittance were determined by numerical minimization [6]. With the only constraint that the dispersion be zero in the insertion device straight sections, the minimum emittance was found to be $69 \mathrm{~nm}^{*} \mathrm{rad}$ at $2.584 \mathrm{GeV}$. When the dispersion is not constrained in the insertion straight sections, the minimum emittance is $21 \mathrm{~nm}^{*} \mathrm{rad}$. The increase in horizontal beam size in the insertion straight sections due to dispersion is more than offset by the reduction in emittance, so the beam size is still reduced in the insertion straights as well as everywhere else in such a lattice (see figure 4 , for example). The optics for the $21 \mathrm{~nm}^{*}$ rad lattice, however, would require huge sextupole strengths which result in a very small dynamic aperture.

\section{B. Minimum practical emittance}

Although the minimum-emittance, $21 \mathrm{~nm}^{*}$ rad lattice has far too small a dynamic aperture, a dynamic aperture as large or slightly larger than the physical aperture can be achieved with a lattice with a $38 \mathrm{~nm} *$ rad emittance. Beam was stored in such a lattice during studies, but the maximum current was only $50 \mathrm{~mA}$ and was limited by the head-tail instability. The present sextupoles in the X-Ray ring are not strong enough to compensate the chromaticity of the $38 \mathrm{~nm}^{*}$ rad lattice. The iron in the verticalchromaticity-compensating sextupoles is already in saturation in the present operations lattice, and the $38 \mathrm{~nm}^{*} \mathrm{rad}$ lattice would require 60 percent stronger sextupoles. To store full current in the $38 \mathrm{~nm}^{*}$ rad lattice would require new sextupole magnets or a feedback system to damp the head-tail instability.

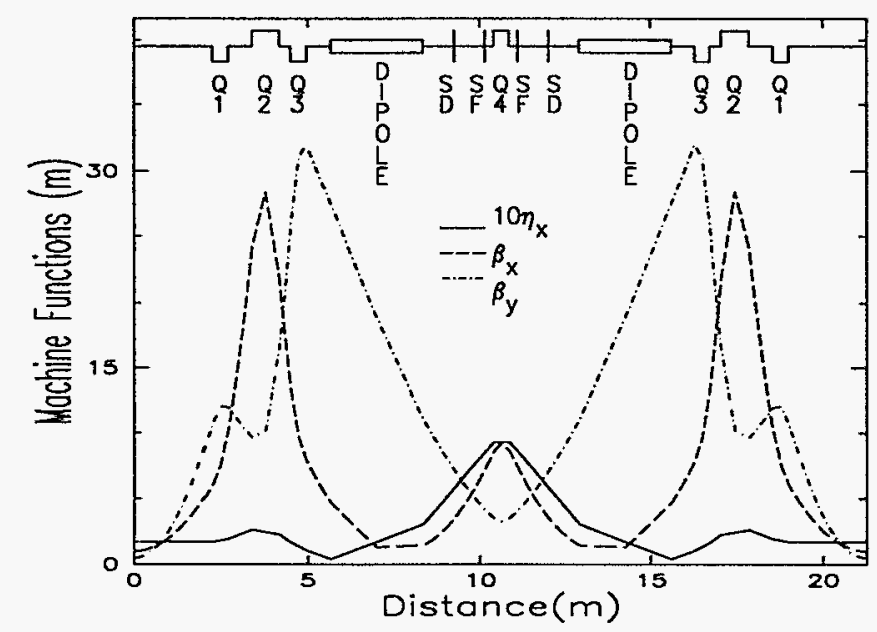

Figure. 3. The optics in the $49 \mathrm{~nm}^{*}$ rad lattice for $1 / 8^{\text {th }}$ of the X-Ray Ring. The four families of quadrupole and two sextupole families are shown.
With the limitation that the vertical-chromaticitycompensating sextupole strengths cannot be increased, the horizontal emittance of the X-Ray Ring can still be decreased by more than a factor of two to $49 \mathrm{~nm}^{*}$ rad. Figure 3 shows the optics for one superperiod (1/8th) of the ring in this lattice. The tunes of this lattice are $9.70\left(\nu_{x}\right)$ and $5.45\left(\nu_{y}\right)$. The dynamic aperture in this lattice is larger than the physical aperture. The reduction in beam size for this $49 \mathrm{~nm}^{*}$ rad lattice compared to the present operations lattice is shown in figure 4 .

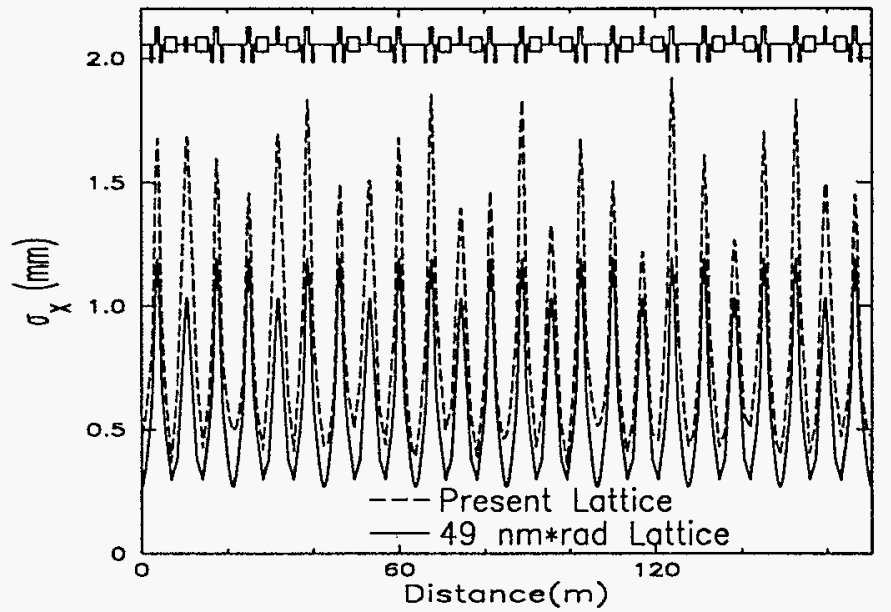

Figure. 4. The horizontal beam size in the $49 \mathrm{~nm}^{*}$ rad lattice compared to that in the present operations lattice $\left(113 \mathrm{~nm}^{*}\right.$ rad $)$ of the X-Ray Ring.

\section{EXPERIMENTAL RESULTS}

As shown in equation 1, the horizontal emittance is determined by the dispersion in the dipole magnets, so controlling the dispersion is critical for achieving low emittance. The work in reference [3] shows that the primary source of the distortion of the measured dispersion (figure 1 ) is the focussing in the sextupoles due to horizontal orbit distortions. The easiest way to correct for this would be to steer the electron beam in the sextupoles. All the synchrotron radiation users, however, are aligned to the present electron orbit, so changing the orbit is not a viable option. Instead, we added trim power supplies to each of the Q4 quadrupoles, and we adjusted the strength of the Q4's to compensate for the beta-weighted integrated focussing strength associated with the four sextupoles surrounding each $\mathrm{Q} 4$.

Figure 2 shows the the improved periodicity of this corrected dispersion. Correcting the dispersion alone reduced the emittance from $113 \mathrm{~nm}^{*}$ rad to $71 \mathrm{~nm}^{*}$ rad. We were able to store the full current of $250 \mathrm{~mA}$ in 25 bunches and $100 \mathrm{~mA}$ in 1 bunch in this $71 \mathrm{~nm}^{*}$ rad lattice. The emittance was measured at $69 \mathrm{~nm}^{*}$ rad by imaging the synchrotron radiation from a dipole magnet using an $x$-ray pinhole camera. The measured $69 \mathrm{~nm}^{*}$ rad agrees with the expected $71 \mathrm{~nm}^{*}$ rad to within measurement accuracy. 


\section{DISCLAIMER}

Portions of this document may be illegible in electronic image products. Images are produced from the best available original document. 
Once the dispersion distortions are removed, further reduction in the emittance can be achieved by adjusting quadrupole family strengths. No attempt has been made yet to store beam in the $49 \mathrm{~nm}^{*}$ rad lattice shown in figure 3. The $49 \mathrm{~nm}^{*}$ rad lattice has tunes that differ from the present operational tunes of $\nu_{x}=9.14$ and $\nu_{y}=6.20$, so

storing beam in the $49 \mathrm{~nm}^{*}$ rad lattice will require setting up a new injection configuration. A current of $50 \mathrm{~mA}$, however, was stored in the $38 \mathrm{~nm}^{*}$ rad lattice, which has tunes approximately the same as the present operations lattice. The expected reduction in beam size in the $38 \mathrm{~nm}^{*}$ rad lattice was confirmed by imaging synchrotron radiation.

\section{CONCLUSIONS}

We have shown that we can control the X-Ray Ring optics and reduce the emittance. We have stored full current in a lattice in which the emittance has been reduced from $113 \mathrm{~nm}^{*}$ rad to $71 \mathrm{~nm}^{*} \mathrm{rad}$. In the future, we will work to store beam in the $49 \mathrm{~nm}^{*}$ rad lattice and explore the potential of this lattice for operations.

\section{ACKNOWLEDGEMENTS}

I would like to thank Sam Krinsky for stimulating discussions. Thanks go to Om Singh and the NSLS power supply group for providing and helping with the Q4 trim power supplies. Also, I would like to thank Julie Leader for her fine work editing this paper.

\section{References}

[1] J. Murphy, Synchrotron Light Source Data Book, BNL 42333, 1993.

[2] J. Safranek and M.J. Lee, "Calibration of the X-Ray Ring Quadrupoles, BPMs, and Orbit Correctors Using the Measured Orbit Response Matrix," AIP Conference Proceedings, Vol 315, 1994.

[3] J. Safranek, Experimental Determination of Linear Optics Including Quadrupole Rotations, these proceedings.

[4] J. Safranek and S. Krinsky, Plans to Increase Source Brightness of NSLS X-Ray Ring, proceedings of the 1993 Particle Accelerator Conference, pg 1491.

[5] M. Sands, SLAC-121, November, 1970.

[6] W. Press, B. Flannery, S. Teukolsky, W. Vetterling, Numerical Recipes, Cambridge, 1990.

\section{DISCLAIMER}

This report was prepared as an account of work sponsored by an agency of the United States Government. Neither the United States Government nor any agency thereof, nor any of their employees, makes any warranty, express or implied, or assumes any legal liability or responsibility for the accuracy, completeness, or usefulness of any information, apparatus, product, or process disclosed, or represents that its use would not infringe privately owned rights. Reference herein to any specific commercial product, process, or service by trade name, trademark, manufacturer, or otherwise does not necessarily constitute or imply its endorsement, recommendation, or favoring by the United States Government or any agency thereof. The views and opinions of authors expressed herein do not necessarily state or reflect those of the United States Government or any agency thereof. 